\title{
The perception of length on curved and flat surfaces
}

\author{
J. FARLEY NORMAN \\ Western Kentucky University, Bowling Green, Kentucky \\ JOSEPH S. LAPPIN \\ Vanderbilt University, Nashville, Tennessee \\ and \\ HIDEKO F. NORMAN \\ Western Kentucky University, Bowling Green, Kentucky
}

\begin{abstract}
In three experiments, observers judged the apparent extents of spatial intervals along the surface of a curved cylinder or a flat plane that was binocularly viewed in a natural, indoor environment. The observers' judgments of surface lengths were precise and reliable but were also inaccurate and subject to relatively large constant errors. These distortions differed among the observers, but they tended to perceive lengths oriented along the curved dimension of the cylinder as being longer than physically equivalent lengths in the noncurved dimension. This phenomenon did not occur when the observers judged curved and noncurved paths on the flat surface. In addition, some observers' judgments of length were affected by changes in the distance to the cylinder, whereas others were affected by the cylinder's orientation in space. These results demonstrate that the perception of length on surfaces is highly dependent on the particular context in which the length occurs.
\end{abstract}

Vision is primarily a system for perceiving spatial relations in the observer's environment. Its effectiveness is sufficient enough that most observers never question how it is achieved. Scientific investigations of spatial vision were begun more than 160 years ago, but many of its basic characteristics remain poorly understood. The perception of length is a good example.

Pioneering psychophysical studies of the perception of length were conducted by Weber in the 1830 s. Weber found that observers could precisely discriminate small differences in the two-dimensional (2-D) lengths of line segments, with discrimination thresholds on the order of $1 \%$ $5 \%$ (Weber, 1834/1978, p. 105). Not long afterward, Fechner studied discriminations of lengths between endpoints of compasses, Volkmann examined perceived distances between parallel threads, and both verified Weber's findings about the precision of length discriminations (reported in Fechner, 1860/1966, pp. 181-183). Weber, Fechner, and other 19th-century investigators also discovered that the perception of length is relative: A just discriminable in-

We thank Myron Braunstein, Hal Sedgwick, and G. John Andersen for their helpful comments and suggestions regarding these experiments. We especially thank Myron Braunstein and John Andersen for suggesting a number of the manipulations included in Experiment 3, which allowed us to separate the relative effects of changes in curvature and depth on the perception of length on real surfaces. Correspondence concerning this article should be addressed to J. F. Norman, Department of Psychology, Western Kentucky University, Bowling Green, KY 42101 (e-mail: farley.norman@wku.edu). crease or decrease in length is an approximately constant proportion, independent of the absolute length (e.g., Weber, 1834/1978, pp. 220-222). Much of the relevant research on length perception in both the 19th and the 20th centuries has been summarized by Baird (1970) and by J. F. Norman, Todd, Perotti, and Tittle (1996).

Many investigators have found that high levels of precision do not occur in all situations. For example, the ability to discriminate which of two lines is longer is good when the two lines are parallel but diminishes when the two orientations differ (Helmholtz, 1866/1925, pp. 174175; see also J. F. Norman et al., 1996). Constant errors and other systematic distortions in perceived length also occur, especially when the lengths have different orientations. The horizontal-vertical illusion is a well-known example: Vertical lengths tend to appear greater than physically equivalent horizontal lengths (Helmholtz, 1866/ 1925 , p. 170; Wundt, $1892 / 1901$, p. 156). Wundt tried to explain this perceived difference in length in terms of the relative ease of eye movements in various directions. Armstrong and Marks (1997) found more recently, however, that the horizontal-vertical illusion is malleable and context dependent, ranging from $3 \%$ to $15 \%$ across conditions.

In a recent experiment using computer-generated displays with lengths defined by both motion and stereoscopic disparities, J. F. Norman et al. (1996) found that length discriminations deteriorated both when the compared lengths had different orientations in three-dimensional (3-D) space and when they were located at different distances from the observer. Average Weber fractions varied 
from $3.3 \%$ to $4.6 \%$ to $13.0 \%$ to $23.2 \%$, depending on whether the lengths were parallel in the frontoparallel plane, different in 2-D orientation in the frontoparallel plane, different in 3-D orientation at the same viewing distance, or different in 3-D orientation and viewing distance, respectively.

The ability to perceive length and spatial extent does not necessarily improve when observers view real objects. For example, Baird and Biersdorf (1967) found that judged extents in depth of cardboard strips placed flat on a table top decreased by $21 \%$ as the strips were moved farther from the observers, from the front to the rear of the table (see also Thouless, 1931). Frisby, Buckley, and Duke (1996) recently conducted an interesting study of length discriminations, using real "gnarled" sticks, and found that when the two sticks had different orientations and different distances (Experiment 6), the Weber fraction for binocular viewing averaged $10.6 \%$. Although this Weber fraction appears at first to be much lower than the $23 \%$ value found by J. F. Norman et al. (1996), the Weber fractions of individual observers in the Frisby et al. study ranged from $4.8 \%$ to $20.1 \%$, and half were above $12.0 \%$. Moreover, the test sticks in the Frisby et al. study were restricted to only two possible 3-D orientations, and this restriction probably led to higher performance than in the comparable conditions of the study by J. F. Norman et al., where the orientations of the two lengths to be compared varied randomly over a wide range and were different on every trial. In any case, from the limited data currently available, the Weber fractions for natural binocular discriminations of lengths of real objects at different orientations and distances appear to be about an order of magnitude worse than those for comparisons of lengths of parallel lines under the best viewing conditions.

Most of the time, human perception is not concerned with line segments, wires, or sticks but, rather, takes place in an environment with extended surfaces, such as the ground plane. One might think that the perception of length or distance might be more accurate (little constant error) and precise (small variability over time) in more ecologically valid situations with the real-world viewing of extended surfaces. But this is not necessarily the case. Gilinsky (1951), Harway (1963), Wagner (1985), and Loomis, Da Silva, Fujita, and Fukusima (1992) all found that distances or lengths in depth appeared to their observers as increasingly smaller and compressed at farther and farther distances. Gilinsky's experiment took place in an indoor archery range, whereas the experiments of Harway, Wagner, and Loomis et al. were conducted outdoors in grassy fields. Physically equivalent lengths in depth did not appear equal. J. F. Norman et al. (1996) required observers to estimate frontoparallel and in-depth lengths between marked locations on a rumpled 3-D surface and also found compressions of the perceived length of indepth intervals. This occurred despite the availability of stereoscopic disparities and motion parallax--the entire experiment was conducted on a table top, all of which was located within $1.8 \mathrm{~m}$ of the observer.
The preceding abbreviated literature review clearly shows that there is no simple answer to fundamental questions about how human observers perceive lengths and distances in an environment, whether simulated or real. In some situations, observers are highly sensitive to differences in lengths, with little constant error, whereas other circumstances lead to poor discriminability and relatively high constant errors. The use of real-world stimuli does not necessarily lead to high levels of performance, and high performance can be obtained under the right conditions in the laboratory.

There is one important situation, however, that has apparently not been systematically examined by perceptual researchers, and that concerns the perception of length or distance along the surface of a single curved object. Previous research on real-world stimuli has used gnarled sticks (Frisby et al., 1996) or relatively flat ground planes or flat table tops (Baird \& Biersdorf, 1967; Gilinsky, 1951; Harway, 1963; Loomis et al., 1992; Thouless, 1931; Wagner, 1985). It is important to keep in mind, however, that most of the time, human observers are perceiving 3-D objects placed against backgrounds. Most of these objects have extended, curved surfaces. It is possible that the perception of length will be more accurate and precise if it takes place within the confines of a single object's surface. The purpose of our experiments was to investigate this possibility by thoroughly examining the perception of lengths along the surface of a real, physically curved object. Since changes in the orientation of a spatial extent often affect its apparent length and changes in the position and orientation of a surface often influence its perceived depth and curvature (see, e.g., Baird \& Biersdorf, 1967; J. F. Norman \& Lappin, 1992; Rogers \& Graham, 1983), we investigated the effects of these manipulations as well, to see whether they influence the perception of length in a more natural viewing context. If observers can perceive length accurately and precisely under any conditions, it should occur with the direct binocular viewing of spatial intervals on real object surfaces at close range in a natural indoor environment where a multiplicity of visual sources of information about 3-D form are available.

\section{EXPERIMENT 1}

The first experiment attempted to assess the accuracy and precision of the perceptions of length on real surfaces by requiring observers to make multiple, repeated judgments about length intervals between separated points on a physical object's surface that was viewed at two different positions in depth. The object's surface was textured and was viewed in an ordinary indoor well-lit environment with many sources of information (supporting table, other objects, etc.) to indicate its position and placement relative to the observer.

\section{Method}

Apparatus. The endpoints of the lengths to be judged by the observers were demarcated by light-emitting diodes (LEDs) that were 
embedded within the surface of the object. The LEDs were controlled by a Silicon Graphics 4D/25 TG workstation using a specially constructed electronic interface, described earlier (J. F. Norman et al., 1996). The SGI workstation was also used to collect the observers' responses.

Stimulus displays. The experimental stimuli were surface lengths defined by the distances along a horizontally oriented cylinder (approximately $28 \mathrm{~cm}$ in diameter) between pairs of LEDs. It is important to keep in mind that these distances along the surface are different from (and longer than) the Euclidean lengths in empty space between the LEDs. The surface of the cylinder was covered with a homogeneous texture pattern resembling white granite. Twenty-four positions covering the frontmost part of the cylinder's surface were chosen purely at random. Therefore, there were 276 lengths that could potentially be investigated ( 24 things taken 2 at a time). From these 276 possible lengths, 30 lengths were chosen for inclusion in the experiment. The chosen lengths fell into three categories: (1) curved in depth, along the curvature of the cylinder (11 lengths), (2) essentially frontoparallel and horizontal, along the noncurved dimension of the cylinder (9 lengths), and (3) obliquely oriented, which had both frontal and in-depth components ( 10 lengths). Since a cylinder has zero Gaussian curvature, it is possible to draw the relative positions of the LEDs on a flat surface-accordingly, the arrangement of the LEDs (from 1 to 24) are shown in Figure 1. A photograph of the cylinder is shown in Figure 2.

As can be seen in Figure 2, the viewing situation is full cue. The surface and its constituent lengths were defined by a myriad of both binocular and monocular cues (stereoscopic disparities, texture gradients, shading, convergence, accommodation, accommodative blur, etc.). The observers used a chinrest to prevent head movements. Lateral head movements might allow the observers to see a partial side

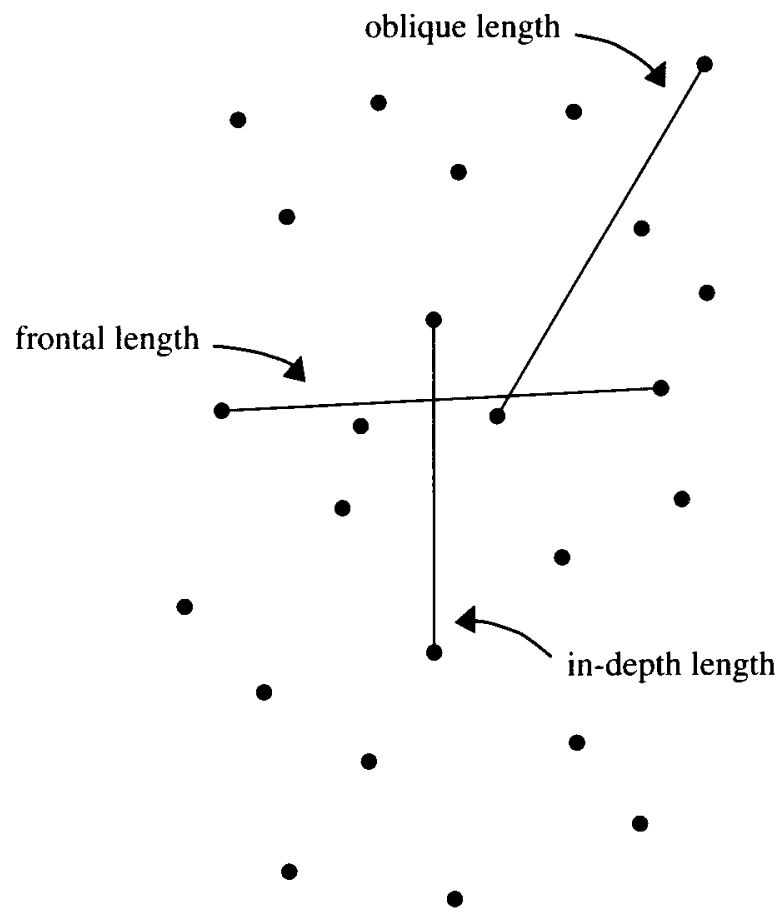

Figure 1. A schematic illustration showing the relative positions of the 24 light-emitting diodes covering the surface of the cylinder. Three representative surface lengths - frontal, in depth, and oblique - are illustrated. The top and bottom of the figure coincide with the curvature of the cylinder, while left and right in the figure represent the noncurved dimension. view of the object and be able to estimate the radius of curvature and so forth. Therefore, head movements were prohibited. All other sources of information normally available in an ordinary environment were present. The cylinder was viewed (in separate blocks of trials) at two different distances from the observer: The front surface was located at an extremely close $50 \mathrm{~cm}$ from the observer's eye or at a farther distance of $180 \mathrm{~cm}$. Both distances are within the near amount of space within which stereopsis is able to effectively operate.

Procedure. Each of 5 observers participated in five experimental sessions for each of the two viewing distances. Three observers judged the stimulus lengths at the far distance first, whereas the remaining 2 observers judged lengths presented at the near distance first. In each session, the observers viewed all of the 30 stimulus lengths twice, in random order. At the completion of the experiment, the observers had estimated each stimulus length 10 times at both viewing distances. On any given trial, the computer would highlight a single pair of LEDs. The observer would then adjust the length of a line segment presented on the SGI monitor with the workstation's mouse until its length matched the apparent length of the distance interval along the cylinder's surface. The adjustable line segment on the SGI monitor was always oriented at an oblique angle of $45^{\circ}$; the monitor itself was always located at a constant, intermediate distance of $100 \mathrm{~cm}$. The observers had an unlimited time to make their judgments. When satisfied with their estimate of the surface length, they pressed a button on the mouse to proceed to the next trial.

Observers. The observers included all three authors (J.F.N., J.S.L., and H.F.N.) and two other observers (C.P.M. and R.N.B.) who were naive with regards to the purposes of the experiment. C.P.M. and R.N.B. had never before participated in a psychophysical experiment.

\section{Results and Discussion}

The results for all 5 observers demonstrate that there is a close relationship between the actual lengths depicted on the cylinder's surface and the observers' perceptions of those lengths. This was true at both of the distances tested, 50 and $180 \mathrm{~cm}$. Figure 3 shows a scatterplot of the actual versus adjusted lengths for one of the naive observers, C.P.M. For this observer, the overall correlation between the actual and the perceived lengths was .968 for the near distance and .950 for the far distance. However, it is evident from an inspection of Figure 3 that there are different relationships for the in-depth, oblique, and frontal lengths. In particular, the regression lines for the in-depth lengths have the highest slopes, followed by the oblique lengths and, finally, the frontal lengths, which have the lowest slopes. These differences in slope were typical: For all observers at both distances, the slopes were higher for the in-depth lengths than for the frontal lengths. These results are shown in Tables 1 and 2. To test whether the observed differences in slope were statistically significant, $t$ tests were performed, following the methods of Howell (1982). All the differences in slope for the near distance were significant, indicating that the lengths oriented in depth along the curvature of the cylinder were perceived as longer than physically equivalent lengths that had frontal orientations along the cylinder's noncurved dimension. This overestimation of in-depth lengths was also significant for 3 of the 5 observers at the far distance.

This conclusion, that in-depth lengths were perceived as longer than frontal lengths, is also evident when the adjustments for individual lengths are considered. For ex- 


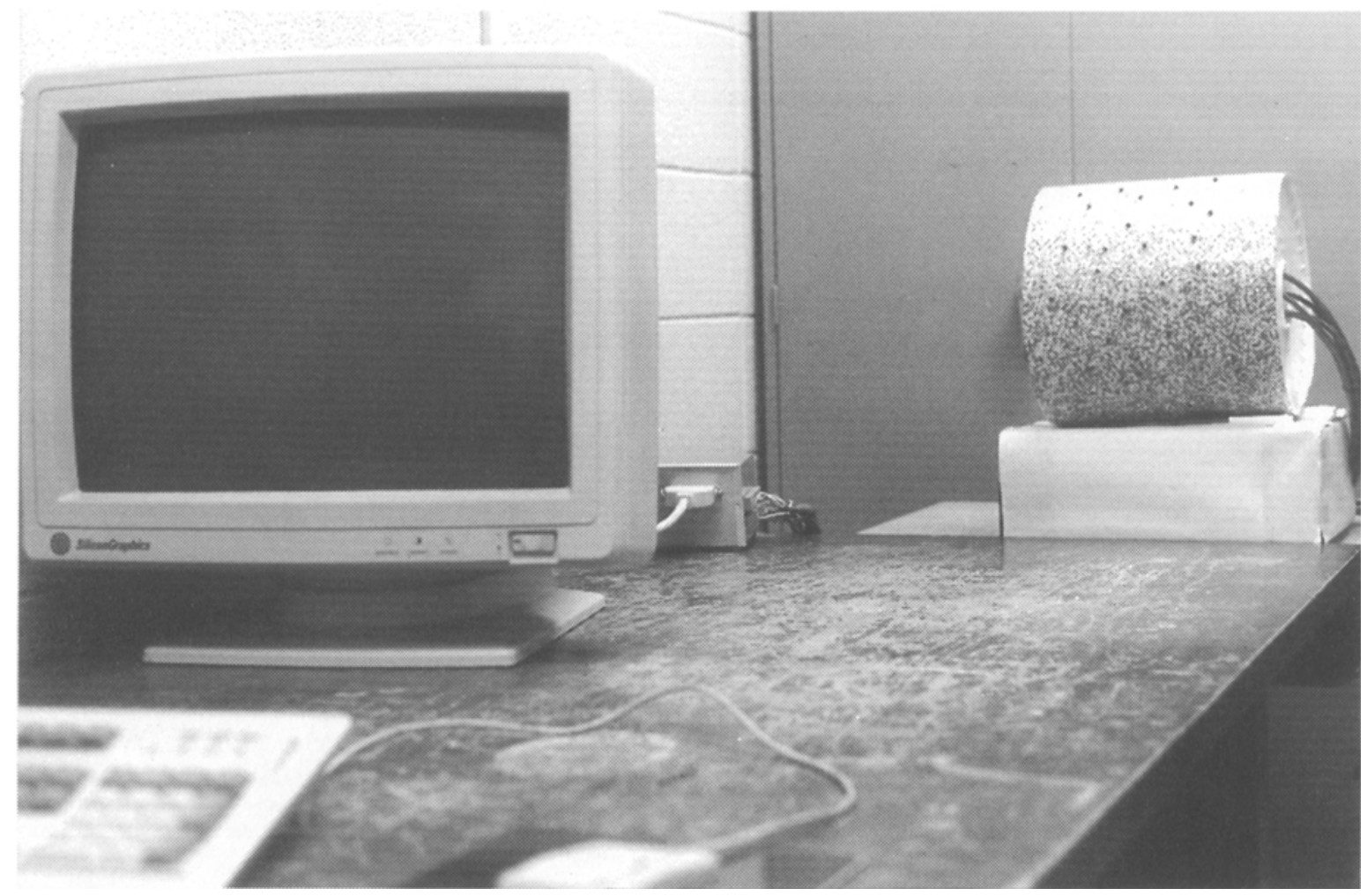

Figure 2. A photograph taken slightly from the side of the observers' vantage point, showing the placement of the cylinder (far viewing distance) and the location of the SGI monitor used to make the matching adjustments.

ample, there were two LED pairs that both had a physical length of $11.0 \mathrm{~cm}$, but all 5 observers at both viewing distances perceived the length between the in-depth pair to be longer - on average, this length was perceived to be $17 \%$ longer than the physically identical frontal length. The uniformity of this result across observers, both experienced and inexperienced, was remarkable. There were some quantitative differences in how the surface lengths were perceived, but all the observers showed essentially the same pattern of results, especially at the near viewing distance.

In contrast to the uniformity in how the in-depth, oblique, and frontal lengths were perceived, the effects of viewing distance were different for different observers. The slopes for the best-fitting regression lines are shown in Table 3 for the near and far distances. Two observers (C.P.M. and J.S.L.) perceived the surface lengths to be significantly longer at the far distance, but the opposite was true for Observers J.F.N. and H.F.N. Observer R.N.B. perceived the lengths to be similar at both distances.

The high correlations between the actual and the perceived lengths for Observer C.P.M. that are evident in Figure 3 also existed for all of the other observers-in fact, the average correlation for individual conditions for all observers was .969. One would then expect that the observers' judgments were reliable, that they exhibited little variability over time. To test this explicitly, we measured the observers' reliabilities as the standard deviation of the 10 adjustments for each condition divided by their mean. The average reliability over all conditions and observers was $7.7 \%$ of the mean, with a standard deviation of 2.9 . Thus, the reliabilities were generally $5 \%-10 \%$ of the mean and were similar in magnitude to those obtained by J. F. Norman et al. (1996). Three of the observers (C.P.M., J.S.L., and H.F.N.) were more reliable at the near distance and somewhat less reliable at the far distance (as a group, reliabilities of $5.8 \%$ vs. $8.6 \%$ for the near and far distances, respectively). In contrast, there were no differences in the reliabilities for the in-depth, oblique, and frontal lengths for any of the observers. The judgments for the in-depth lengths oriented along the curvature of the cylinder were just as reliable as those for the frontally oriented lengths.

The preceding analysis showed that the amount of variability across repeated judgments is reasonably low, so there is not much variable error. But what about constant error? How much did the observers' judgments vary from the correct values? To answer this question, we calculated constant errors for each of the 30 lengths on the cylinder's surface according to the following equation. It measures how much the observers' 10 judged lengths for each condition deviate from the actual length: 


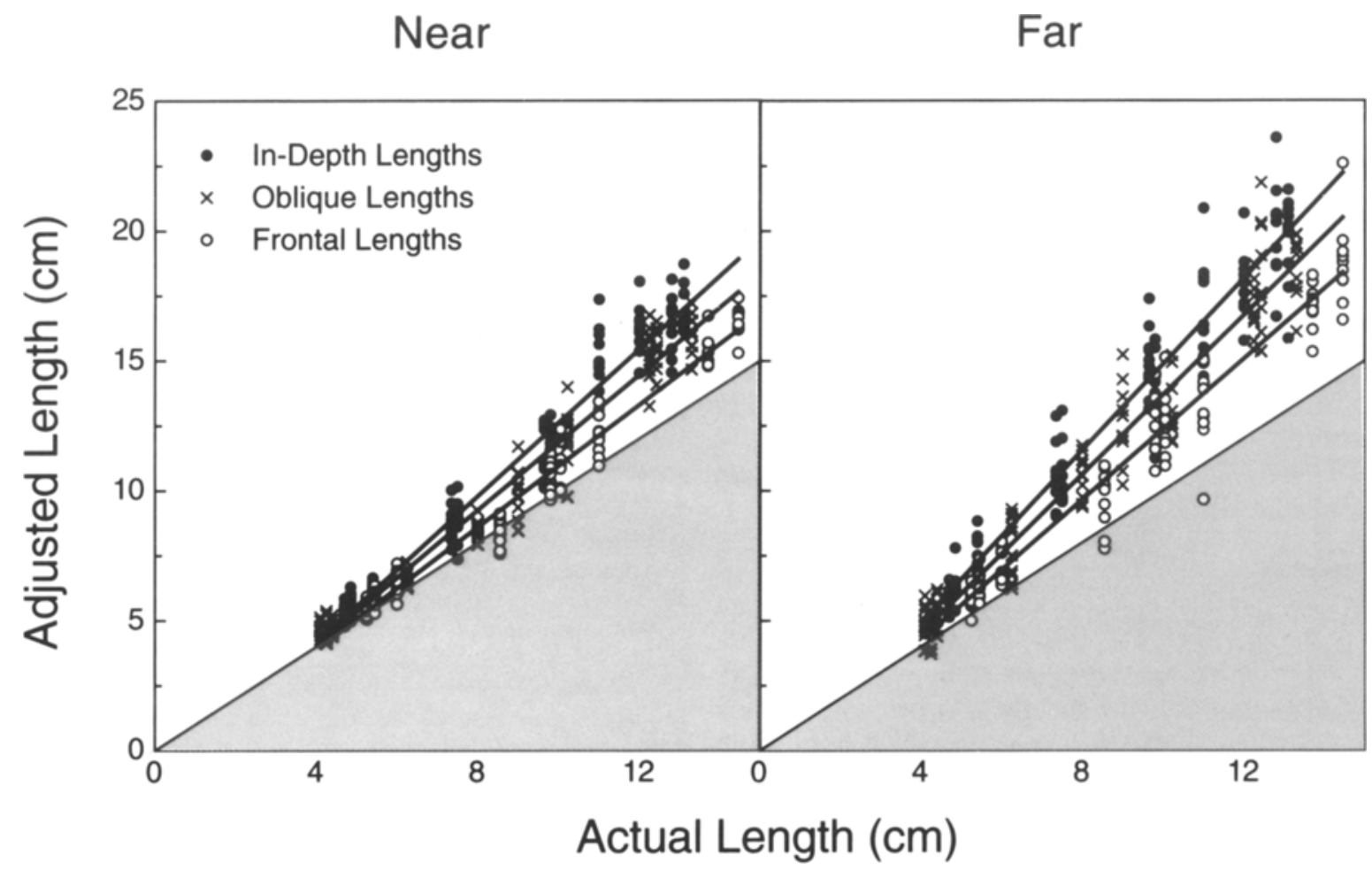

Figure 3. Results of Experiment 1 for observer C.P.M. The abscissa indicates the actual surface lengths, whereas the ordinate indicates the observer's adjusted lengths. The results for the in-depth, frontal, and oblique surface lengths are plotted separately. The best-fitting regression lines are also shown. The line separating the shaded and unshaded portions of the plots represents the identity line, where the adjusted lengths equal the actual lengths. Therefore, the shaded area indicates the lengths that were perceived to be shorter than the actual lengths, whereas the unshaded area indicates the lengths that were perceived to be longer than they actually were.

$$
\text { Constant error }=\frac{\sum_{i=1}^{10} \mid \text { adjusted }- \text { actual } \mid}{\text { actual }} \text { - }
$$

For each observer, the individual errors for each of the 30 surface lengths at the near distance were averaged together to produce an overall error value. The same procedure was used to calculate a constant error value for the far distance results. Figure 4 shows these average constant errors, along with the reliabilities for comparison. The magnitudes of the constant errors vary widely, from a low of $8.4 \%$ of the correct values to a high of $46.5 \%$. This latter magnitude would indicate that the observers' perceived lengths deviated on average (in the near condition, $\mathrm{Ob}$ server H.F.N.) from the actual lengths by $46.5 \%$. It is clear that for some observers at some distances, the constant errors were reasonably low $(8.4 \%-15.2 \%)$, but for other observers and distances, the constant errors were very high, indicating the presence of large perceptual distortions (i.e., large deviations from the correct values). Three of the observers (C.P.M., J.S.L., and R.N.B.) exhibited more veridical judgments (lower errors) at the near viewing distance, whereas Observers J.F.N. and H.F.N. were more veridical at the far viewing distance.
Figure 5 illustrates how the observers' constant errors vary as a function of the orientation of the lengths on the cylinder's surface. Once again, the reliabilities for the same conditions are plotted for comparison. All 5 observers' constant errors are lowest for the frontal lengths $(7.0 \%-27.8 \%)$ and are generally highest for the in-depth lengths $(14.0 \%-50.4 \%)$, with the oblique error values generally falling in between $(13.4 \%-47.8 \%)$. It is interesting that these large perceptual distortions of the indepth and oblique lengths were always perceptual expansions, not compressions. The perceived magnitudes of these lengths were always similar to or longer than the correct values (i.e., the slopes of the best-fitting regression

Table 1

Results of the Regression Analysis Conducted on the Results Obtained at the Near Viewing Distance in Experiment 1

\begin{tabular}{|c|c|c|c|c|}
\hline \multirow[b]{2}{*}{ Observer } & \multicolumn{2}{|c|}{ Slope } & \multirow[b]{2}{*}{$t$} & \multirow[b]{2}{*}{$p$} \\
\hline & In-Depth Lengths & Frontal Lengths & & \\
\hline C.P.M. & $1.41 \overline{3}$ & 1.175 & $\overline{5.989}$ & $<.001$ \\
\hline J.S.L. & 1.250 & 1.106 & 3.637 & $<.001$ \\
\hline J.F.N. & 1.150 & 0.956 & 5.827 & $<.001$ \\
\hline H.F.N. & 1.373 & 1.286 & 2.437 & .016 \\
\hline R.N.B. & 1.258 & 0.995 & 4.570 & $<.001$ \\
\hline
\end{tabular}

Note-All $d f=196$. 
Table 2

Results of the Regression Analysis Conducted on the Results Obtained at the Far Viewing Distance in Experiment 1

\begin{tabular}{lcccc}
\hline & \multicolumn{3}{c}{ Slope } & \\
Observer & In-Depth Lengths & Frontal Lengths & $t$ & $p$ \\
\hline C.P.M. & 1.654 & 1.354 & 5.207 & $<.001$ \\
J.S.L. & 1.534 & 1.260 & 4.894 & $<.001$ \\
J.F.N. & 1.032 & 0.961 & 2.187 & .030 \\
H.F.N. & 1.317 & 1.250 & 1.487 & .139 \\
R.N.B. & 1.085 & 1.033 & 0.897 & .371 \\
\hline
\end{tabular}

Note-All $d f=196$.

lines for the in-depth lengths in Tables 1 and 2 are all greater than 1.0, never less). It is also important to keep in mind that these perceptual distortions (which were different for the in-depth, oblique, and frontal lengths; see Figure 3 and Tables 1 and 2) all took place within the confines of a single, physical object placed on a tabletop right in front of the observer. The observers were not allowed to touch the cylinder, but they easily could have done so at the near distance $(50 \mathrm{~cm})$, had they wished. At this distance, even small depth differences produce significant binocular disparities. The observers had a wealth of information to support their judgments (stereoscopic disparities, texture gradients, shading, convergence, accomodative blur, etc.); nevertheless, substantive distortions occurred in the observers' perceptions of surface lengths.

\section{EXPERIMENT 2}

The results of the first experiment indicated that there were significant distortions in the observers' perceptions of length that depended on the orientations of the lengths on the cylindrical object's surface. Most of the observers' judgments were also affected by the position of the cylinder in depth. But what about other possible distortions? It has frequently been reported for computer-generated displays that there is an anisotropy of orientation for stereoscopically defined surfaces (Gillam, Chambers, \& Russo, 1988; J. F. Norman \& Todd, 1995; Rogers \& Graham, 1983) and for motion-defined surfaces (Cornilleau-Pérès \& Droulez, 1989; J. F. Norman \& Lappin, 1992; Rogers \& Graham, 1983), so that surface curvatures are more readily perceived and detected when they occur in some directions, but not in others. For example, when viewing

Table 3

Results of the Regression Analysis Comparing the Effects of Viewing Distance in Experiment 1

\begin{tabular}{lccrr}
\multicolumn{5}{c}{ Viewing Distance in Experiment 1 } \\
\cline { 2 - 3 } Observer & Near Judgments & Far Judgments & $t$ & $p$ \\
\hline C.P.M. & 1.288 & 1.502 & -6.227 & $<.001$ \\
J.S.L. & 1.164 & 1.398 & -7.500 & $<.001$ \\
J.F.N. & 1.059 & 1.001 & 2.747 & .006 \\
H.F.N. & 1.332 & 1.251 & 2.670 & .008 \\
R.N.B. & 1.141 & 1.071 & 1.920 & .055 \\
\hline
\end{tabular}

Note-All $d f=596$. stereoscopically defined cylinders or sinusoidal surfaces that are curved in one dimension only, curvatures along a vertical direction (e.g., a horizontal cylinder or a sinusoidal surface with horizontal peaks and troughs) are perceived at lower disparity amplitudes than are equivalent vertical cylinders or vertically oriented sinusoidal surfaces. One purpose of this second experiment was to investigate whether there is any significant anisotropy in the perception of indepth lengths that occurs when observers view physically curved surfaces that have different orientations in space. A related purpose was to determine whether the overestimation of curved lengths in depth relative to frontal lengths found in Experiment 1 depends on the specific direction in which the curvature takes place.

\section{Method}

Apparatus. The apparatus (LEDs, electronic interface, and SGI workstation) was the same as that used in Experiment 1.

Stimulus displays. The experimental stimuli were the same 30 surface lengths that were investigated in Experiment 1 . The only difference was that the previously horizontal cylinder was rotated $90^{\circ}$ so that in Experiment 2, it was oriented vertically. Following this rotation, the previously used in-depth lengths were still oriented in depth, the previous frontal lengths still had frontal orientations, and so forth. Everything was identical-each length could serve as its own control. The only difference between the stimulus displays used in Experiments 1 and 2 was the orientation of the cylinder in space. Only the far viewing distance $(180 \mathrm{~cm})$ was used in this experiment.

Procedure. The procedures used in Experiment 2 were the same as those in Experiment 1. The observers once again participated in five experimental sessions, with two judgments of each of the 30 stimulus lengths in each session. At the conclusion of the experiment, each observer had estimated each surface length 10 times.

Observers. The observers were the same as those who had participated in Experiment 1.

\section{Results and Discussion}

The observers' variable and constant errors are shown in Figure 6. Once again, the observers were reliable, with standard deviations of $10 \%$ of the mean or less. Generally, however, their judgments were not accurate. The constant errors ranged from $6.3 \%$ to $51.3 \%$, depending on the observer and condition, reflecting relatively large distortions in the observers' perceptions of length.

As can be seen from an inspection of Table 4, the change in orientation of the cylinder in space had large effects on the perception of in-depth lengths for some observers, but not for others. In particular, there were significant reductions in the slopes of the best-fitting regression lines for Observers C.P.M., J.S.L., and J.F.N. However, this did not occur for Observers H.F.N. and R.N.B. Their perceptions of the in-depth lengths remained essentially unchanged. There were also differences in how the frontal lengths were perceived following the change in orientation of the cylinder. Figures 7 and 8 compare the results of Experiments 1 and 2 for Observers J.S.L. and H.F.N. One can see that J.S.L.'s judgments of the frontal lengths were not affected by the orientation of the cylinder but that the judgments of H.F.N. were markedly af- 


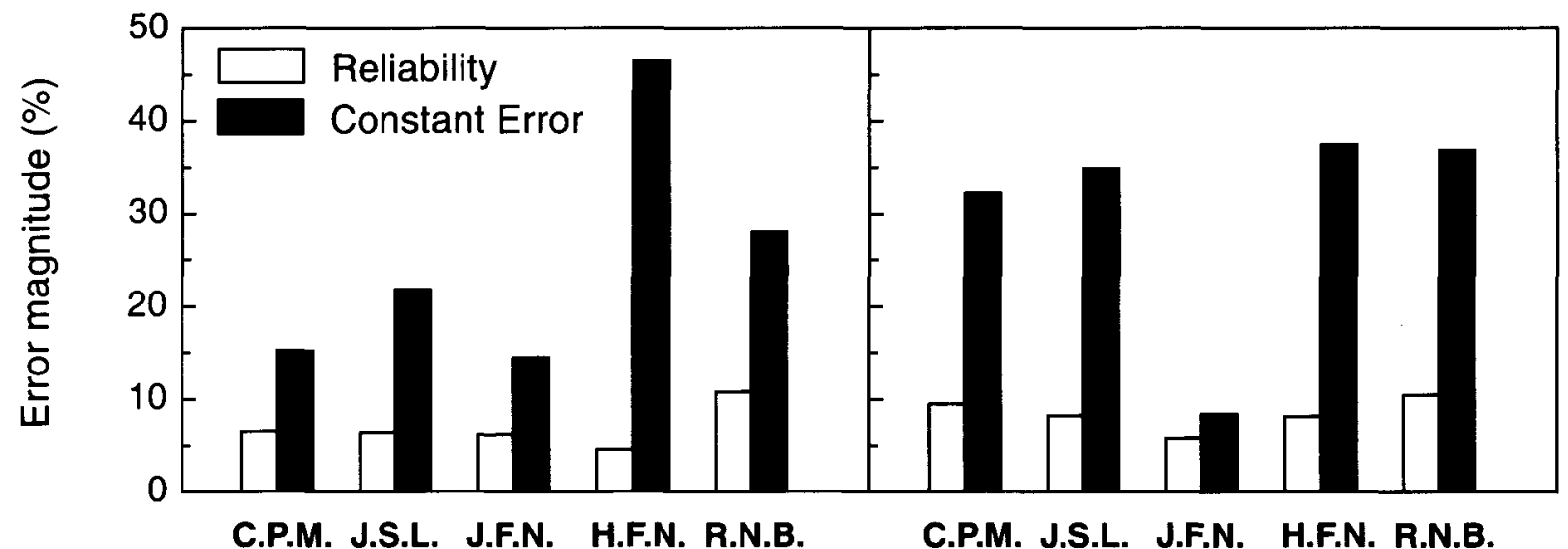

Figure 4. The observers' constant errors in Experiment 1, plotted as a function of viewing distance. The observers' reliabilities for the same conditions are also indicated for comparison. Lower reliability values indicate that the observers were more reliable (i.e., lower variability).

fected. For this observer, the frontal lengths appeared longer when they were vertically oriented, as is characteristic of the horizontal-vertical illusion.

The change in orientation of the cylinder from horizontal to vertical led to other effects as well. These are shown in Table 5. Because of the reductions in the slopes of the in-depth lengths for Observers C.P.M., J.S.L., and J.F.N. (see Table 4), the previous difference between the in-depth and the frontal lengths found in Experiment 1 either disappeared (C.P.M. and J.F.N.) or was reversed

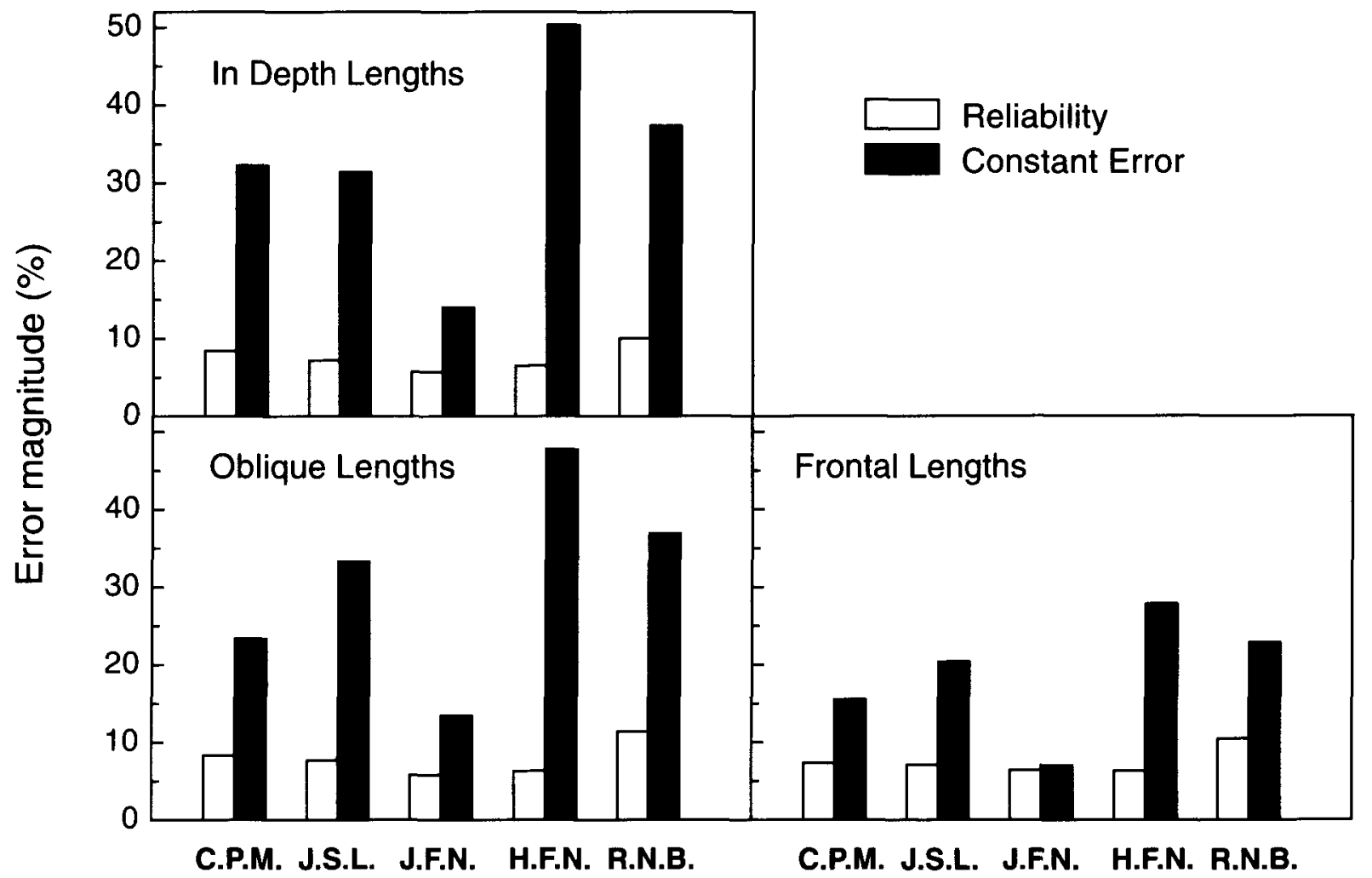

Figure 5. The observers' constant errors in Experiment 1, plotted as a function of surface length orientation. The observers' reliabilities for the same conditions are also indicated for comparison. 


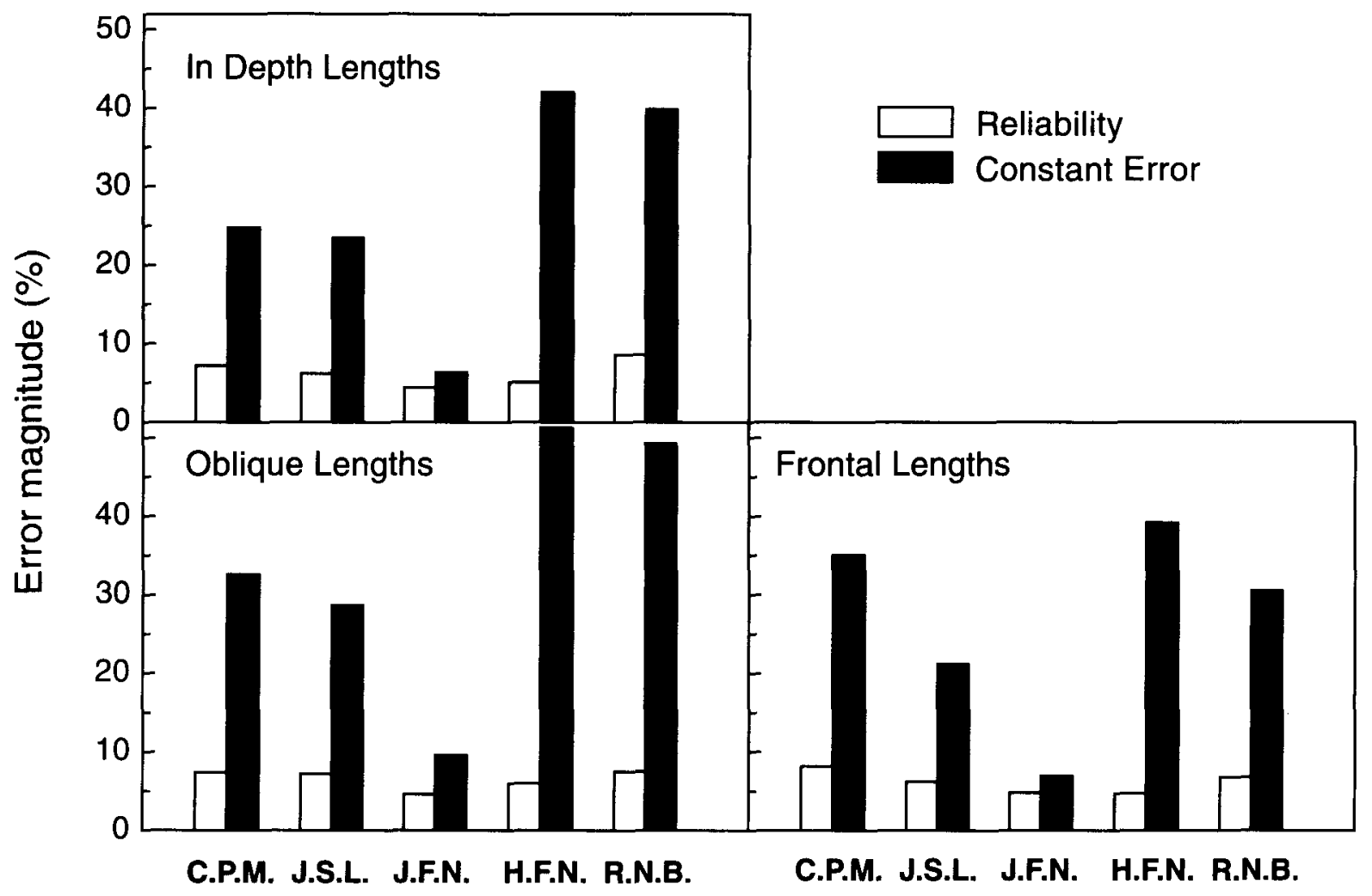

Figure 6. Results of the reliability and constant error analysis on the results of Experiment 2, plotted as a function of the lengths' orientation on the cylindrical surface.

(J.S.L.). However, for the remaining 2 observers, who did not show a significant effect of surface length orientation at the far distance in Experiment 1 (H.F.N. and R.N.B.), they did show the effect at the far distance in Experiment 2 , when the cylinder's orientation was vertical. Thus, the results of Experiment 2 fall into two groups. One group (C.P.M., J.S.L., and J.F.N.), who previously showed a significant overestimation of in-depth lengths relative to frontal lengths when the cylinder was horizontally oriented, did not do so when the cylinder's orientation was changed to vertical. Exactly the opposite pattern held for the remaining group of observers (H.F.N. and R.N.B.): These observers showed the overestimation when the cylinder was vertically oriented, but not when it was horizontally oriented.

Table 4

Results of the Regression Analysis Comparing the In-Depth Results of Experiments 1 and 2

\begin{tabular}{lccrrr}
\hline & \multicolumn{5}{c}{ Slope In-Depth Lengths } \\
Observer & Horizontal Cylinder & Vertical Cylinder & & \multicolumn{1}{c}{$t$} & \multicolumn{1}{c}{$p$} \\
\hline C.P.M. & 1.654 & 1.279 & 7.124 & $<.001$ \\
J.S.L. & 1.534 & 1.184 & 6.273 & $<.001$ \\
J.F.N. & 1.032 & 0.872 & 6.179 & $<.001$ \\
H.F.N. & 1.317 & 1.324 & -0.179 & .858 \\
R.N.B. & 1.085 & 1.166 & -1.522 & .129 \\
\hline
\end{tabular}

Note-All $d f=216$.

\section{EXPERIMENT 3}

One of the most prominent findings of the previous two experiments is that lengths along the curvature of the cylinder tended to be perceived as longer than physically equivalent lengths along the noncurved dimension of the cylinder. This effect was consistent at the near distance in Experiment 1 but depended on the cylinder's orientation (i.e., horizontal or vertical) when it was viewed at the far distance. However, in all cases in Experiments 1 and 2, the cylinder was oriented so that the lengths associated with its curvature were extended in depth away from the observers, whereas the lengths along the noncurved dimension of the cylinder were essentially frontoparallel. Therefore, from the evidence presented so far, it is impossible to determine whether the observed perceptual distortions are related to how the lengths are oriented, relative to the directions of curvature along the cylinder's surface, or whether the distortions occurred because some lengths were extended in depth whereas others were not. The purpose of this experiment was to resolve this ambiguity by separating the effects of curvature and changes in depth on the perception of length.

\section{Method}

Apparatus. The same electronic interface was used to turn on and off the LEDs defining the surface extents to be judged as that 

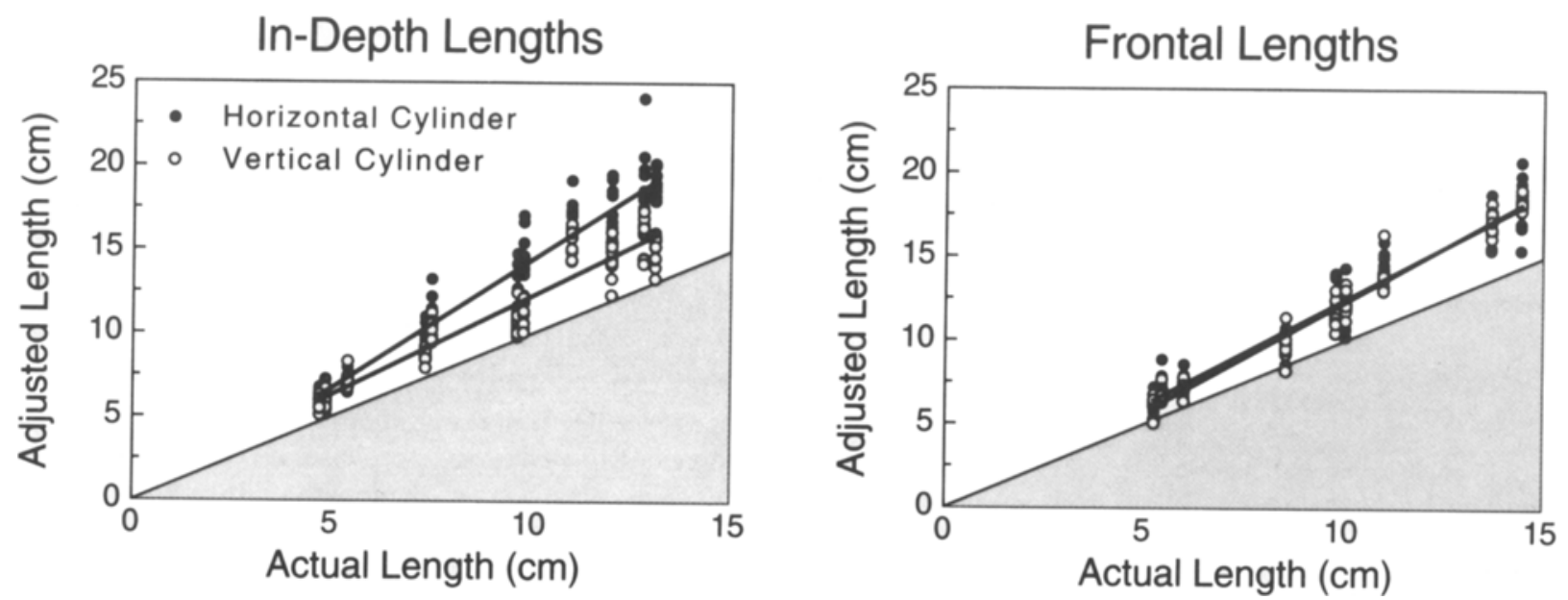

Figure 7. A comparison of the results of Experiments 1 and 2 for observer J.S.L. Separate comparisons are plotted for the in-depth lengths (left panel) and the frontal lengths (right panel). The filled circles indicate the results of Experiment 1 , where the cylinder's orientation was horizontal, whereas the open circles show the analogous results for Experiment 2, where the orientation of the cylinder was vertical. The solid curves illustrate the best-fitting regression lines for each condition. The line separating the shaded and the unshaded portions of the plots represents the identity line, where the adjusted lengths equal the actual lengths. Therefore, the shaded area indicates the lengths that were perceived to be shorter than the actual lengths, whereas the unshaded area indicates the lengths that were perceived to be longer than they actually were.

used in Experiments 1 and 2. However, for this experiment, the electronic interface was controlled by an Atari 1040ST computer, whereas an Apple Power Macintosh 8600/300 was used to record the observers' responses. The adjustable lengths used to match the apparent extents of the spatial intervals on the surfaces were presented by the PowerMacintosh using a Mitsubishi 91TXM 21-in. monitor.

Stimulus displays. The experimental stimuli were surface lengths defined by the distances along either the same cylinder that had been used in Experiments 1 and 2 or a flat planar surface covered with the same granite texture as the cylinder. The cylinder was placed in either of two orientations: (1) the same as that used in Experiment 1 (horizontal orientation), where the lengths along the noncurved dimension were essentially frontoparallel, or (2) a slanted orientation, so that the previously frontoparallel noncurved lengths were now extended in depth (i.e., this configuration differed from that of Experiment 1 only by a $45^{\circ}$ rotation about a Cartesian verti- cal axis). The flat planar surface was oriented so that it was essentially frontoparallel. Ten lengths were chosen on both the cylinder and the plane for the observers to estimate. These lengths fell into two categories, curved and noncurved. Thus, there were six qualitatively different contexts in which the lengths appeared: (1) curved only in depth (cylinder, no slant), (2) curved with both in-depth and frontal components (cylinder, $45^{\circ}$ slant), (3) curved only in the frontoparallel plane (flat planar surface), (4) noncurved in the frontoparallel plane on a curved surface (cylinder, no slant), (5) noncurved, extended in depth (cylinder, $45^{\circ}$ slant), and (6) noncurved in the frontoparallel plane on a flat surface. The radius of curvature of the curved lengths on the flat planar surface was the same as the radius of curvature of the cylinder, $28 \mathrm{~cm}$.

Procedure. The procedures used were the same as those in Experiments 1 and 2 . The observers made their estimates of surface length by using the mouse on the PowerMacintosh to match an ad-
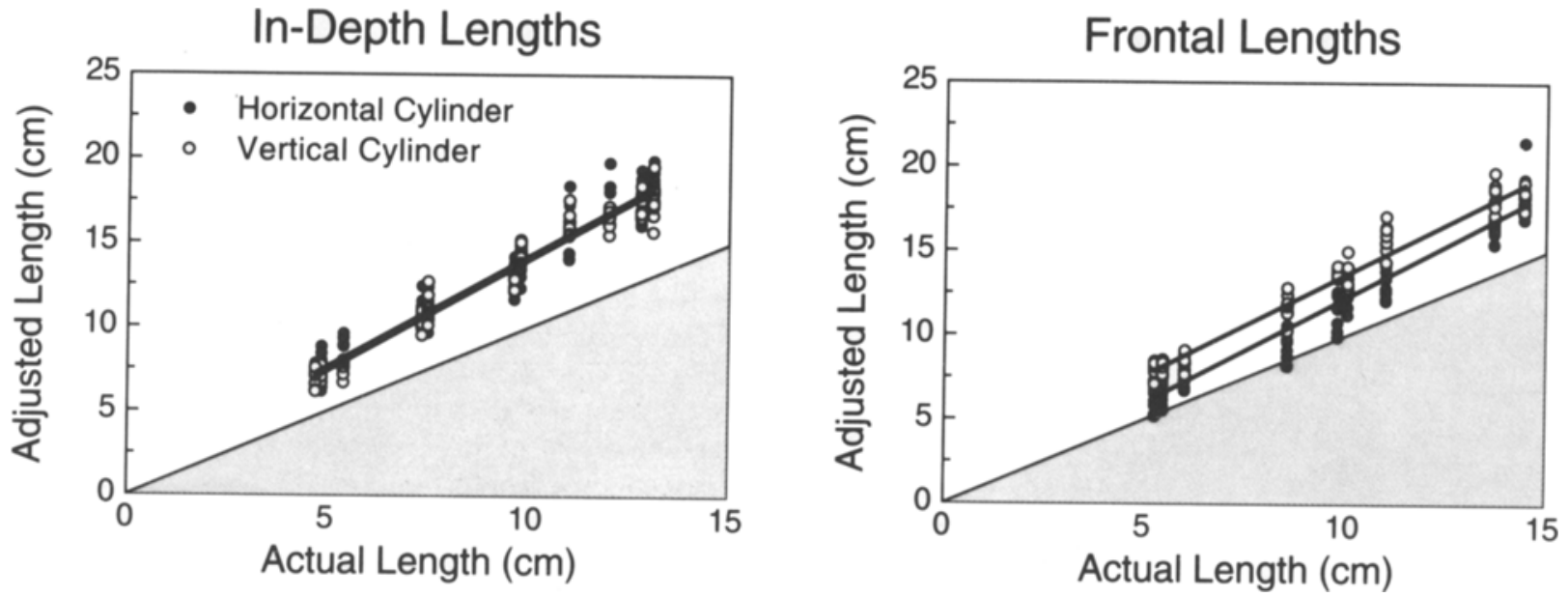

Figure 8. A comparison of the results of Experiments 1 and 2 for Observer H.F.N. 
Table 5

Results of the Regression Analysis Conducted on the Results Obtained in Experiment 2

\begin{tabular}{lccrrr}
\hline & \multicolumn{3}{c}{ Slope } & & \\
\cline { 2 - 3 } Observer & In-Depth Lengths & Frontal Lengths & \multicolumn{1}{c}{$t$} & \multicolumn{1}{c}{$p$} \\
\hline C.P.M. & 1.279 & 1.220 & 1.303 & .194 \\
J.S.L. & 1.184 & 1.318 & -3.023 & .003 \\
J.F.N. & 0.872 & 0.864 & 0.382 & .703 \\
H.F.N. & 1.324 & 1.206 & 3.373 & $<.001$ \\
R.N.B. & 1.166 & 1.039 & 2.902 & .004 \\
\hline
\end{tabular}

Note-All $d f=196$.

justable length to that of a length highlighted on the cylinder's or the plane's surface. Separate blocks of trials were used for the three different surface conditions (frontal cylinder, slanted cylinder, and flat plane). Within each block, the observers estimated the 10 lengths ( 5 curved +5 noncurved) 10 times in a randomly determined order. The surface lengths were viewed by the observers from the far viewing distance, $180 \mathrm{~cm}$.

Observers. The observers included 1 observer who had previously participated in Experiments 1 and 2 (J.S.L.) and a new naive observer, who had never before performed the surface length adjustment task (T.E.D.).

\section{Results and Discussion}

The slopes of the best-fitting regression lines between the actual and the adjusted lengths for the 2 observers are shown in Figures 9 and 10 . Once again, the observers' judgments were precise. In particular, the mean correlation coefficient was .976 for judging lengths on the cylinder's surface, whereas the average correlation was .988 for the judgments of length along the flat planar surface. The results for the frontal cylinder condition replicated the findings of Experiment 1 for Observer J.S.L., and the effect of the orientation of the length on the cylinder's surface was even stronger for the new Observer, T.E.D. From an inspection of Figure 9, it is apparent that the overestimation of curved lengths relative to noncurved lengths is limited to situations in which observers are judging lengths along the surface of a curved object. This effect did not exist when the observers estimated lengths on a physically
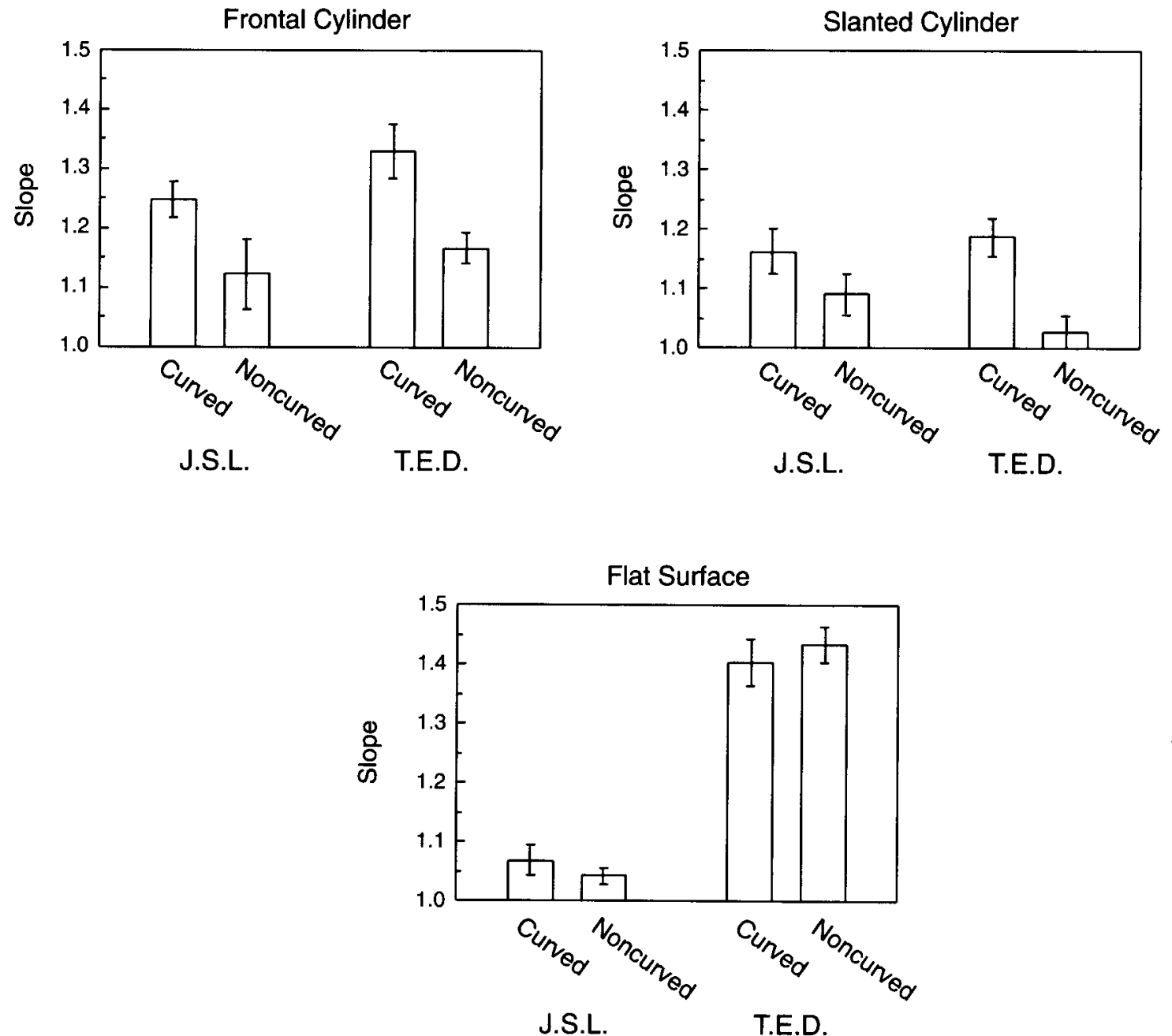

Figure 9. The results of Experiment 3 for Observers J.S.L. and T.E.D. The slopes of the best-fitting regression lines between actual and adjusted lengths are plotted for each of the conditions used in Experiment 3 . The error bars indicate \pm one standard error. 
J.S.L.

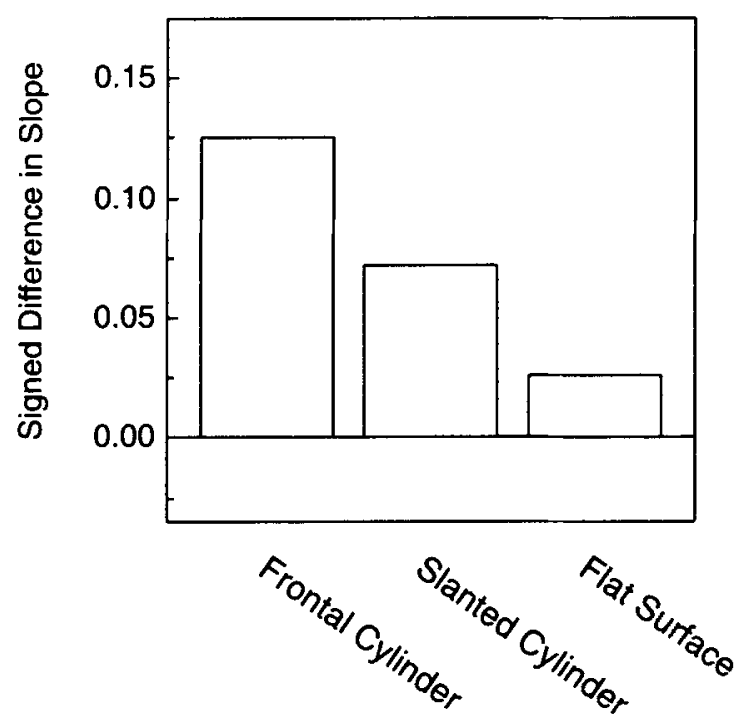

T.E.D.

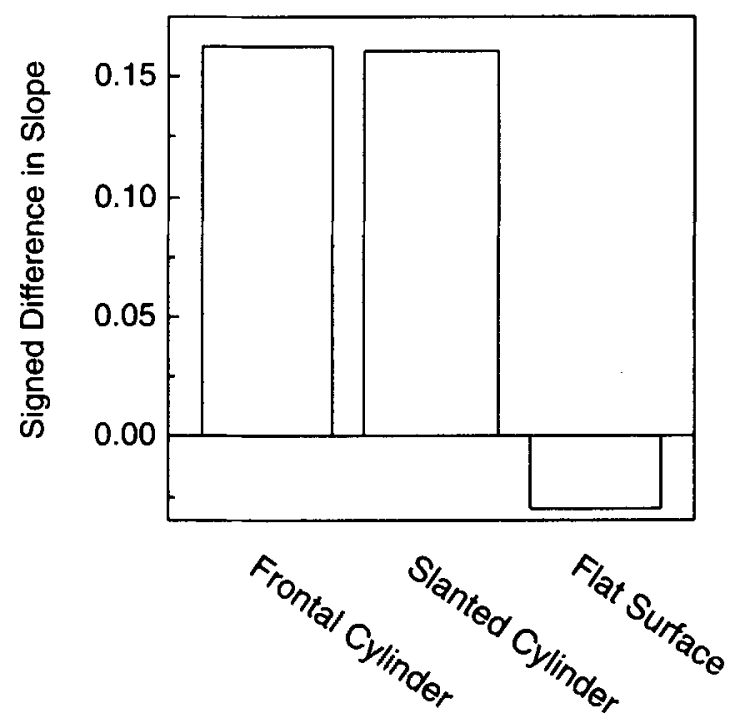

Figure 10. The results of Experiment 3, showing that the overestimation of curved lengths relative to noncurved lengths is eliminated when observers judge lengths on flat surfaces. The overestimation effect is limited to curvatures in depth.

flat surface (see Figure 10). Neither is a simple change in depth sufficient to cause high slopes of regression lines or large overestimations. Notice in Figure 9 that the pattern of results when the cylinder was slanted in depth by $45^{\circ}$ is similar to that observed when the cylinder was frontal but is reduced in magnitude. Once again, the curved lengths were perceived as longer than the noncurved lengths, even though the noncurved lengths were now extended in depth. It is important to note that the slopes for the noncurved lengths extended in depth are smaller than those for the noncurved frontoparallel lengths in the frontal cylinder condition; therefore, simple extension of the length in depth cannot be the cause of the basic overestimation effect observed in Experiments I and 2. Apparently, the difference in perceived length between differently oriented surface extents occurs only when observers are judging lengths on a surface that is curved in depth.

One other finding that is evident in the results shown in Figure 9 is the large overestimation of all lengths, both curved and noncurved, for Observer T.E.D. that occurred while judging lengths on the flat planar surface. Such overestimation of frontoparallel lengths at far viewing distances has been found in earlier experiments (e.g., Baird \& Biersdorf, 1967; J. F. Norman et al., 1996, see Figure 10). It is an interesting fact that this large overestimation did not take place when Observer T.E.D. judged frontoparallel lengths on the cylinder at the same distance; his judgments were more veridical on the curved surface than on the flat surface.

\section{GENERAL DISCUSSION}

The results of all three experiments clearly demonstrate that human observers can make reasonably precise judg- ments about the lengths along smoothly curved and flat surfaces but that their judgments are often inaccurate and beset with large constant errors. As a group, the observers' average correlation in Experiment 1 between the actual and the perceived lengths for single conditions was .969; that indicates that fully $94 \%$ of the variance in the observers' perceived lengths can be accounted for by the variability within the original stimulus lengths. In contrast, the mean constant error was $27.6 \%$ in Experiment 1 and $29.4 \%$ in Experiment 2, reflecting the fact that the observers' perceptions of length deviated, on average, more than $25 \%$ from the actual lengths. This pattern, that the observers may have been reliable but not accurate, closely resembles that found by J. F. Norman et al. (1996). That study found that the observers' perceptions of 3-D length in empty space between surface locations were reasonably reliable (standard deviations were $5.1 \%-10.6 \%$ of the mean) but inaccurate (constant errors ranged from $10.0 \%$ to $39.7 \%$ ).

One purpose for conducting the experiments discussed here was to examine whether the perception of lengths would be more accurate if they were not straight-line distances in space but occurred along the surface of a physical object. One might hypothesize that the perception of length might be more accurate in this latter, more ecologically valid context. The results of the present experiments suggest that the answer is no-humans do not necessarily perceive lengths along a surface more accurately than they do the distances or lengths in space between objects or between separate locations on a single object. Our finding of small reliability values (i.e., moderate to high precision) and large constant errors occurs for other types of visual judgments as well. Consider, for example, the perception of speed within a moving pattern. McKee (1981) has shown 
that observers can precisely discriminate differences in the speed of moving patterns (Weber fractions of $4 \%-8 \%$ ), whereas the results of Brown (1931) and H. F. Norman, J. F. Norman, Todd, and Lindsey (1996) indicate that the perceived speed of any given moving pattern can be highly influenced by the context within which the motion is placed. Similarly, in our present experiments, the perception of surface length depended on such factors as (1) the viewing distance to the object (Experiment 1), (2) the type of object, flat or curved (Experiment 3), (3) the physical orientation of the object in space (Experiments 1 and 2 ), and (4) whether the length took place along a straight or a curved path on the surface itself (Experiments 1,2, and 3 ).

The effects of the orientation of the stimulus length on the cylinder's surface were particularly robust and occurred for all the observers at the near distance $(50 \mathrm{~cm})$ in Experiment 1 and for most of the observers at the far distance $(180 \mathrm{~cm})$. Surface lengths that were oriented along the curvature of the cylinder in depth were perceived to be significantly longer than physically equivalent lengths that were not curved in depth. This effect was also present in Experiments 2 and 3, depending on the particular observer and the experimental condition. It is interesting that this type of distortion involving curved lengths in depth is different than the compressive distortion that has typically been found in previous research on the perception of depth intervals (e.g., Baird \& Biersdorf, 1967; Gilinsky, 1951; Harway, 1963; Loomis et al., 1992; J. F. Norman et al., 1996; Wagner, 1985). This previous research, conducted using real-world physical stimuli, documented that there is often a compressive distortion that takes place when viewing depth intervals, so that any given depth interval appears smaller and smaller in extent as the depth interval is viewed from farther and farther distances. This can be seen on a daily basis - the yellow or white stripes separating adjacent lanes on a highway can be meters long but do not often appear that large when seen at a distance while driving. This effect is accentuated if one looks farther and farther ahead along the roadway. The distortions of length found in the present set of experiments occurred within the confines of a single curved object (i.e., the cylinder) and appear to be different than the distortions previously discussed in the literature. The probable reason this distortion was not noticed previously is that it appears to be limited to situations in which observers are estimating lengths on a surface with a significant amount of curvature; it was not evident in our experiments when the observers made similar judgments on a flat planar surface. The studies cited above (e.g., Baird \& Biersdorf, 1967; Loomis et al., 1992) required observers to estimate the magnitude of extents in depth on a flat surface (e.g., a flat table or a ground plane).

Finally, the results of the present experiments show that the anisotropy of orientation that has been demonstrated numerous times both for stereopsis and structure-frommotion in experiments using computer-generated displays (Cornilleau-Pérès \& Droulez, 1989; Gillam et al., 1988;
J. F. Norman \& Lappin, 1992; J. F. Norman \& Todd, 1995; Rogers \& Graham, 1983) may also occur when curved surfaces are viewed in the physical environment. In the past, stereoscopic surfaces with curvature in a vertical direction (e.g., a horizontally oriented cylinder) were easier to detect and discriminate than surfaces with curvatures in a horizontal direction (e.g., vertical cylinders). The same pattern of results was found for surfaces defined by structure-from-motion (see, e.g., J. F. Norman \& Lappin, 1992) when the axis of rotation was vertical. An analogous result was found in the present experiments for 3 of the 5 observers: In-depth lengths along the curvature of the cylinder were perceived to be longer when the cylinder was horizontal and were perceived to be shorter when the cylinder was rotated to a vertical orientation.

The perception of length is apparently a complicated process involving a wide variety of factors. Perceived lengths evidently are not related in any simple way to actual physical lengths (see also Rubin, 1950, for especially strong distortions in perceived 2-D lengths). The particular relationship, or distortion, that is exhibited depends on whether the interval to be judged occurs in empty space between environmental locations (e.g., J. F. Norman et al., 1996) or along a continuous surface (Experiments 1, 2, and 3); if on a surface, the perceived length depends on the type, placement, and orientation of the surface relative to an observer. Finally, different patterns often emerge for each individual observer. However, for each observer, the relationships are lawful, in that the observed relationship is stable and reliable across multiple experimental sessions that are days apart (informal observations in our laboratory suggest that these relationships are stable for weeks, if not for months or years). This variability in how spatial relationships are perceived for different observers in a physical environmental setting has been noted before (Battro, Netto, \& Rozestraten, 1976). More research will evidently be needed to fully understand why individual observers perceive length differently in natural settings.

\section{REFERENCES}

Armstrong, L., \& Marks, L. E. (1997). Differential effects of stimulus context on perceived length: Implications for the horizontal-vertical illusion. Perception \& Psychophysics, 59, 1200-1213.

BaIRD, J. C. (1970). Psychophysical analysis of visual space. New York: Pergamon.

BAIRD, J. C., \& BIERSDORF, W. R. (1967). Quantitative functions for size and distance judgments. Perception \& Psychophysics, 2, 161-166.

Battro, A. M., Netto, S. P., \& Rozestraten, R. J. A. (1976). Riemannian geometries of variable curvature in visual space: Visual alleys, horopters, and triangles in big open fields. Perception, 5, 9-23.

Brown, J. F. (1931). The visual perception of velocity. Psychologische Forschung, 14, 199-232.

Cornilleau-Pérès, V., \& Droulez, J. (1989). Visual perception of surface curvature: Psychophysics of curvature detection induced by motion parallax. Perception \& Psychophysics, 46, 351-364.

Fechner, G. T. (1966), Elements of psychophysics (Vol. 1; H. E. Adler. Trans.). New York: Holt, Rinehart \& Winston. (Original work published 1860)

Frisby, J. P., BuCkLEY, D., \& DUKE, P. A. (1996). Evidence for good recovery of lengths of real objects seen with natural stereo viewing. Perception, 25, 129-154. 
GILINSKy, A. S. (1951). Perceived size and distance in visual space. Psychological Review, 58, 460-482.

Glllam, B., Chambers, D., \& Russo, T. (1988). Postfusional latency in stereoscopic slant perception and the primitives of stereopsis. Journal of Experimental Psychology: Human Perception \& Performance, 14, 163-175.

HARWAY, N. I. (1963). Judgment of distance in children and adults. Journal of Experimental Psychology, 65, 385-390.

Helmholtz, H. (1925). Helmholtz's treatise on physiological optics (Vol. 3; J. P. C. Southall, Trans.). Menasha, WI: Optical Society of America. (Original work published 1866)

HowelL, D. C. (1982). Statistical methods for psychology. Boston: Duxbury.

Loomis, J. M., Da Silva, J. A., Fujita, N., \& Fukusima, S. S. (1992). Visual space perception and visually directed action. Journal of Experimental Psychology: Human Perception \& Performance, 18, 906921.

MCKeE, S. P. (1981). A local mechanism for differential velocity detection. Vision Research, 21, 491-500.

Norman, H. F., Norman, J. F., Todd, J. T., \& Lindsey, D. T. (1996). Spatial interactions in perceived speed. Perception, 25, 815-830.

Norman, J. F., \& LapPIN, J. S. (1992). The detection of surface curvatures defined by optical motion. Perception \& Psychophysics, 51, 386396.
Norman, J. F., \& ToDD, J. T. (1995). The perception of 3-D structure from contradictory optical patterns. Perception \& Psychophysics, 57, 826-834.

Norman, J. F., Todn, J. T., Perotti, V. J., \& Tittle, J. S. (1996). The visual perception of three-dimensional length. Journal of Experimental Psychology: Human Perception \& Performance, 22, 173-186.

Rogers, B. J., \& Graham, M. E. (1983). Anisotropies in the perception of three-dimensional surfaces. Science, 221, 1409-1411.

RubiN, E. (1950). Visual figures apparently incompatible with geometry. Acta Psychologica, 7, 365-387.

Thouless, R. H. (1931). Phenomenal regression to the real object: 1 . British Journal of Psychology, 21, 339-359.

Wagner, M. (1985). The metric of visual space. Perception \& Psychophysics, 38, 483-495.

WeBER, E. H. (1978). The sense of touch (H. E. Ross, Trans.). New York: Academic Press. (Original work published 1834)

WUNDT, W. (1901). Lectures on human and animal psychology (J. E. Creighton \& E. B. Titchener, Trans.). New York: Macmillan. (Original work published 1892)

(Manuscript received January 12, 1999; revision accepted for publication September 10, 1999.) 